\title{
21 School cultural congruity
}

\author{
Mihya Weber, Chloe Zhang, Alessandra \\ Mittelstet, and Shane R. Jimerson
}

\section{How to use this policy}

School cultural congruity refers to the cultural match between the school and the students and families who are served by the school. Elements of cultural (in)congruity exist within all areas of the school environment. For example, differences in school and student or family cultural values may exist in school rules and policies, expectations for students and parents, curriculum, methods of teaching, and approaches to discipline. Nonetheless, establishing a school environment that is culturally congruent with the student population is valuable in promoting positive student academic outcomes (Amatea, 2009). School leaders, teachers, and student support staff are key in developing a culturally congruent school environment and should receive professional development and ongoing support to implement strategies aimed at promoting school cultural congruity. This policy example is intentionally broad and school policy creators are encouraged to delete and build upon suggestions below to create a policy that best represents their school.

[To adapt and use this policy, delete or modify the text as indicated]

\section{[INSERT name of school] School cultural congruity}

\section{Rationale}

The importance of K-12 school climate has been increasingly recognized by researchers and educators. School climate reform is recommended to promote healthy relationships and lower dropout rates among students. School climate impacts students' academic success and socio-emotional wellbeing and can mitigate the negative impact of disadvantaged socioeconomic status. School climate has been identified as a protective factor for the learning and positive life development of students (Thapa et al., 2013). A positive school climate is characterized by having norms, goals, values, interpersonal relationships, teaching and learning practices, and organizational structures that promote people's positive experiences of school life (Thapa et al., 2013). School cultural congruity, which emphasizes a cultural match between the school and 
the students and families served by the school, is one important aspect that reflects the general school climate.

Cultivating a school environment that is culturally congruent with the student population can be valuable in promoting positive student outcomes, particularly through encouraging parent engagement. The importance of parent engagement in children's learning is long established (Oswald et al., 2017), yet many schools struggle to achieve high levels of parental engagement. Christenson and Sheridan (2001) highlight the importance of schools and show how their current infrastructure may be excluding some families. School environments that are exclusionary to some students and families will have negative effects on the learning outcomes of those students. Castellanos and Gloria (2007) define cultural congruity as the fit between the student's and the educational institution's values, which promotes connectedness with the school environment. Christenson and Sheridan (2001) highlight that cultural mismatches between a school and students/families occur when values held sacred in one culture are misunderstood or invalidated in another. This mismatch may be seen across a variety of elements in the school context, including expectations for behaviour, levels of performance, problem-solving, goal setting, and behaviour management methods (Christenson \& Sheridan, 2001). This mismatch between educators and students can have adverse effects on parental engagement efforts and student learning outcomes. By promoting a culturally congruent school environment, students and parents can engage more fully in learning opportunities.

\section{Purpose}

The purpose of this policy is to provide guidance on promoting a school culture that is congruent with the students and families served.

\section{Scope}

This policy applies to school leadership personnel, teachers, and student support staff. School leaders are essential in implementing and supporting staff in school-wide changes. Teachers and support staff will apply elements of establishing a sense of cultural congruity in the classroom with materials used, approaches to learning, and interactions with parents and students.

[INSERT specific roles and responsibilities of individuals or teams]

\section{Policy statement}

Christenson and Sheridan (2001) emphasize that cultural mismatches between a school and students/families occur when values held sacred in one culture are misunderstood or invalidated in another. Accordingly, 
- Staff at [INSERT name of school] recognize that their experiences and cultural values may differ from students and parents.

- School leaders and educators at [INSERT name of school] strive to understand students' and their family's cultural background as it relates to their experiences with school.

- Staff at [INSERT name of school] engage in ongoing professional development to better understand how dominant cultural norms impact how we approach the education of our students.

- Staff engage in ongoing professional development to better understand the cultural norms related to education of students and families with diverse backgrounds at [INSERT name of school].

- Staff at [INSERT name of school] view diversity as an asset to the learning experience of all students and seek to honour cultural differences.

- School leaders at [INSERT name of school] seek consultation with cultural brokers when working with students and families from different cultural backgrounds.

- Staff at [INSERT name of school] embrace feedback and adjust accordingly with an open mind.

Cultural mismatch may be seen across a variety of elements in the school context, including expectations for behaviour, levels of performance, problemsolving, goal setting, and behaviour management methods (Christenson \& Sheridan, 2001). This has implications for school leaders.

- School leaders at [INSERT name of school] seek parent feedback on expectations of behaviour and approaches to discipline should infractions occur.

- School leaders at [INSERT name of school] seek parent feedback on expectations of academic performance and approaches to intervention.

- Educators at [INSERT name of school] seek parent feedback on goals for students and approaches to problem-solving when goals are not met.

- Educators at [INSERT name of school] seek parent feedback on role expectations for parents and educators.

- Educators at [INSERT name of school] engage in ongoing and proactive communications with parents and students and ensure their feedback is considered and implemented.

Christenson and Sheridan (2001) highlight the importance of communicating with parents clearly and in a way that is understandable to them.

- Written communication at [INSERT name of school] is disseminated to parents in their preferred language and mode of communication.

- School staff at [INSERT name of school] engage parents in meetings in a manner that is informative, engaging, and respectful. 
- School leaders at [INSERT name of school] ensure that educators have reliable access to translators and interpreters when communicating with parents who speak a different language.

- School staff at [INSERT name of school] provide ongoing opportunities for parent feedback on how to promote effective and culturally and linguistically appropriate communications.

Amatea (2009) explains that when instruction is only administered from a single perspective and does include diverse approaches, students from different backgrounds are likely to experience increased challenges in achieving positive academic outcomes.

- Educators at [INSERT name of school] incorporate a variety of instructional approaches and methods for evaluating student achievement.

- Educators at [INSERT name of school] utilize course materials that reflect the diversity of the student population.

- Educators at [INSERT name of school] incorporate examples in teaching that are reflective of student experiences.

- Educators at [INSERT name of school] involve the students in the selection and incorporation of reflective materials and examples.

- School leaders at [INSERT name of school] provide consistent support to teachers and support staff to engage in culturally congruent practices with students and families.

- School leaders at [INSERT name of school] provide regular training opportunities for teachers and support staff to improve their cultural competence in teaching.

- School leaders at [INSERT name of school] provide educators with necessary materials to implement culturally relevant assignments and decorations in the classroom.

\section{Authorship}

Mihya Weber, Chloe Zhang, Alessandra Mittelstet, Shane R. Jimerson, University of California Santa Barbara

[INSERT RELEVANT STAFF MEMBERS]

\section{Related policy and documents}

[INSERT RELEVANT POLICY AND DOCUMENTS]

\section{Date of ratification}

This policy was ratified on the [INSERT DATE]. 


\section{Rating of evidence base}

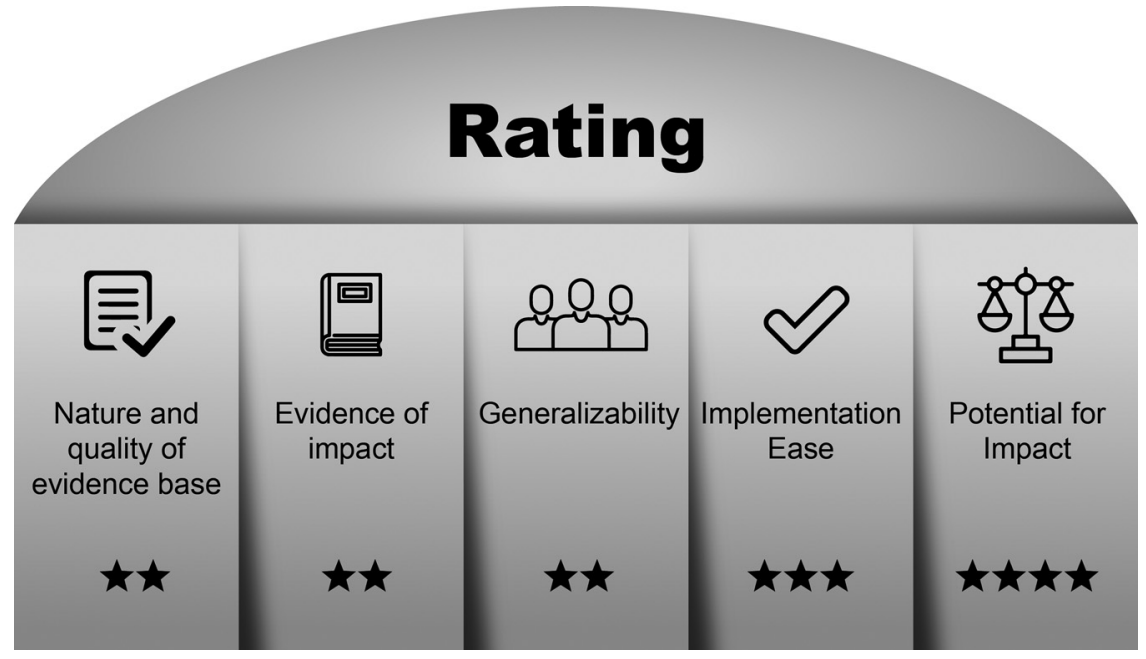

Figure 21.1. School Cultural Congruity Rating of Evidence.

Author note. Robust evidence supports the importance of cultural competence and culturally relevant practices in education. Though school cultural congruity is a new construct to be examined in K-12 education settings, elements of the construct are strongly supported. Positive student outcomes have been extensively documented in higher education but not yet examined in K-12 education settings. However, the impact of culturally relevant practices in K-12 education is long established. Cultural congruity has been extensively investigated in higher education but is new to primary and secondary education settings. However, the value of cultural responsiveness in K-12 education is long established. Most elements of school cultural congruity can be achieved by adapting routine practices. Depending on the discrepancy between the dominant culture and the student population, additional materials (e.g., books that reflect diverse cultures) may be needed to achieve full implementation. Though school cultural congruity is a relatively new construct to be examined in primary and secondary education settings, given the strong evidence of positive student outcomes associated with elements within the construct of cultural congruity, the potentiality for positive results is high.

\section{Date of review}

This policy will be reviewed by [INSERT DATE].

\section{Further reading}

Amatea, E. S. (2009). Building culturally responsive family-school relationships. Boston: Allyn \& Bacon.

Christenson, S., \& Sheridan, S. M. (2001). Schools and families: Creating essential connections for learning. New York: Guilford Press. 
Gay, G. (2000). Culturally responsive teaching: Theory, practice, and research. New York: Teachers College Press.

\section{Expert consultation}

The policy should be informed by school teams focused on diversity, equity, inclusion, and culturally responsive instruction. Comprehensive school safety planning teams could provide positive contributions to developing culturally congruent safety plans and interventions. There would also be value to coordinating with the school administrators to ensure school leaders have resources and support necessary to effectively implement new policies.

\section{References}

Amatea, E. S. (2009). Building culturally responsive family-school relationships. Allyn \& Bacon.

Castellanos, J., \& Gloria, A. M. (2007). Research considerations and theoretical application for best practices in higher education. Journal of Hispanic Higher Education, 6(4), 378-396.

Christenson, S., \& Sheridan, S. M. (2001). Schools and families: Creating essential connections for learning. Guilford Press.

Oswald, D. P., Zaidi, H. B., Cheatham, D. S., \& Brody, K. G. (2017). Correlates of parent involvement in students' learning: Examination of a national data set. Journal of Child and Family Studies, 27(1), 316-323.

Thapa, A., Cohen, J., Guffey, S., \& Higgins-D'Alessandro, A. (2013). A review of school climate research. Review of Educational Research, 83(3), 357-385. 